\title{
APPROXIMATING COMPACT AND WEAKLY COMPACT OPERATORS 1
}

\author{
JOHN W. BRACE
}

TheOREM 1. A linear operator $T$ is compact if and only if it is a cluster point for the topology $\alpha$ of a sequence $\left\{F_{n}\right\}$ of continuous linear operators with finite dimensional range.

REMARKs. To place the theorem in context consider all operators as mapping the Banach space $X$ into the Banach space $Y$ and consider the topology $\alpha$ to be that of almost uniform convergence on the unit ball of $X$ utilizing the norm topology on $Y$. The second theorem gives a similar result for weakly compact operators. All definitions and background information can be found in the two references.

Proof of Theorem 1. Assuming the sequence $\left\{F_{n}\right\}$ to have $T$ as a cluster point, there is a subnet $\left\{F_{\gamma}, \gamma \in G\right\}$ converging to $T$ for the topology $\alpha$. The second adjoint operators $\left\{F_{\gamma}^{* *}, \gamma \in G\right\}$ form a Cauchy net for the topology of almost uniform convergence on the unit ball $S^{* *}$ of $X^{* *}$ utilizing the norm topology on $Y^{* *}[1$, Theorem 4.1]. Define a linear operator $F_{0}$ which agrees with $T^{* *}$ on the image of $X$, while for all other $x^{* *}$ in $X^{* *}, F_{0}\left(x^{* *}\right)=\lim _{\gamma} F_{\gamma}^{* *}\left(x^{* *}\right)$.

Consider an arbitrary positive number $\epsilon$ and a net $\left\{x_{\delta}^{* *}, \delta \in D\right\}$ in the image of $S$ in $X^{* *}$ converging to a point $x_{0}^{* *}$ in $X^{* *}$ for the $X^{*}$ topology. There exist $\delta_{0}$ in $D$ and $\gamma_{1}, \gamma_{2}, \cdots, \gamma_{k}$ in $G$ such that

$$
\begin{gathered}
\min _{i=1,2, \ldots, k}\left\|F_{0}\left(x_{\delta}^{* *}\right)-F_{\gamma_{i}}^{* *}\left(x_{\delta}^{* *}\right)\right\|<\frac{\epsilon}{3} \quad \text { for all } \delta>\delta_{0}, \\
\left\|F_{\gamma_{i}}^{* *}\left(x_{\delta}^{* *}\right)-F_{\gamma_{i}}^{* *}\left(x_{0}^{* *}\right)\right\|<\frac{\epsilon}{3}
\end{gathered}
$$

for $i=1,2, \cdots, k$ and all $\delta>\delta_{0}$, and $\left\|F_{\gamma_{i}}^{* *}\left(x_{0}^{* *}\right)-F_{0}\left(x_{0}^{* *}\right)\right\|<\epsilon / 3$ for $i=1,2, \cdots, k$. Thus $\left\|F_{0}\left(x_{\delta}^{* *}\right)-F_{0}\left(x_{0}^{* *}\right)\right\|<\epsilon$ for all $\delta>\delta_{0}$ and $F_{0}$ is continuous for the $X^{*}$ topology on $S^{* *}$ and the norm topology on $Y^{* *}$. Therefore $F_{0}$ is $T^{* *}$ and $T$ is compact.

For the converse let $\beta$ be the directed set composed of all continuous projections with finite dimensional range in $Y$, the order being determined by the inclusion ordering on their ranges. The net $\left\{P^{* *} T^{* *}, P \in \mathcal{P}\right\}$ converges pointwise to $T^{* *}$ on $S^{* *}$ and a known theorem for continuous functions says that due to the metric topology on $Y^{* *}$ and the compactness of $S^{* *}$ the net can be replaced by a

Received by the editors June 6, 1960.

1 This research was supported by the National Science Foundation, Grant 9414. 
sequence $\left\{P_{n}^{* *} T^{* *}\right\}$ having $T^{* *}$ as a cluster point. ${ }^{2} T^{* *}$ must also be a cluster point of the sequence for the topology of almost uniform convergence on $S^{* *}$ with the norm topology on $Y^{* *}[1$, Theorem 4.2]. Therefore it is concluded that $T$ is a cluster point of the sequence $\left\{P_{n} T\right\}$ for the topology $\alpha$.

By omitting the sequence and resorting to the topology $\beta$, almost uniform convergence on the unit ball of $X$ with the weak topology on $Y$, the same line of reasoning gives Theorem 2 .

THEOREM 2. A linear operator $T$ is weakly compact if and only if it is the limit point for the topology $\beta$ of a net $\left\{F_{\gamma}, \gamma \in G\right\}$ of continuous linear operators with finite dimensional range.

\section{REFERENCES}

1. J. W. Brace, The topology of almost uniform convergence, Pacific J. Math. vol. 9 (1959) pp. 643-652.

2. N. Dunford and J. T. Schwartz, Linear operators, Part I, New York, Interscience, 1958.

\section{UNIVERSITY OF MARYLAND}

2 The author is unable to locate a reference. A version of the theorem appeared at one time in a manuscript copy of J. L. Kelley's book on linear topological spaces. The statement is as follows. "Let $F$ be a subset of the space of continuous functions with compact domain $S$ and range in a metric space. If $f$ is in the closure of $F$ for the topology of pointwise convergence, then there is a sequence in $F$ having $f$ as a cluster point." 